\title{
Influence of Coupled Sidesticks on the Pilot Monitoring's Awareness During Flare
}

\author{
Alan F. Uehara* and Dominik Niedermeier ${ }^{\dagger}$ \\ DLR (German Aerospace Center), Braunschweig, Germany, 38108
}

\begin{abstract}
Passive sidesticks have been used in modern fly-by-wire commercial airplanes since the late 1980s. These passive sidesticks typically do not feature a mechanical coupling between them, so the pilot's and copilot's sidesticks move independently. This characteristic disabled the pilot monitoring (PM) to perceive the control inputs of the pilot flying (PF). This can lead to problems of awareness in abnormal situations. The development of active inceptor technology made it possible to electronically couple two sidesticks emulating a mechanical coupling. This research focuses on the benefits of coupled sidesticks to the situation awareness of the PM. The final approach and landing scenario was considered for this study. Twelve pilots participated in the simulator experiment. Results suggest that the coupling between sidesticks, allowing the PM to perceive the PF's inputs, can improve the PM's situation awareness.
\end{abstract}

\section{Nomenclature}

$\begin{array}{llll}\text { ADI } & \text { Attitude Director Indicator } & \text { PF } & \text { Pilot Flying } \\ \text { AGL } & \text { Above Ground Level } & \text { PFD } & \text { Primary Flight Display } \\ \text { ATC } & \text { Air Traffic Control } & \text { PM } & \text { Pilot Monitoring } \\ \text { DLR } & \text { German Aerospace Center } & \text { PNF } & \text { Pilot Not Flying } \\ \text { FAA } & \text { Federal Aviation Administration } & \text { SA } & \text { Situation Awareness } \\ \text { FBW } & \text { Fly-By-Wire } & \text { SOP } & \text { Standard Operating Procedure } \\ \text { FCS } & \text { Flight Control System } & \text { TOGA } & \text { Takeoff/Go-Around } \\ \text { KCAS } & \text { Knots Calibrated Airspeed } & \text { VMC } & \text { Visual Meteorological Conditions }\end{array}$

\section{Introduction}

$\mathrm{T}$

HE control inceptors and the actuators of the control surfaces are mechanically decoupled in fly-by-wire

(FBW) airplanes. Therefore, the pilots do not have to use their strength to overcome friction, inertia and aerodynamic loads to control the aircraft. This evolution of flight control systems allowed the substitution of the control column by small sidesticks. Among the advantages of using sidesticks are the better visibility of the instrument panel, less complexity of the mechanical assembly, less weight, and simpler maintenance. ${ }^{1}$

The first manufacturer to use sidesticks in commercial FBW airplanes was Airbus with the A320, which entered into service in 1988. Other examples of FBW airplanes with sidesticks are the Dassault 7X and the Embraer Legacy 500/450. The common characteristic of the sidesticks in these airplanes is that they are passive. This means that the handling characteristics of the sidesticks are defined by their internal mechanical components, namely springs and dampers.

\footnotetext{
${ }^{*}$ Guest scientist, Institute of Flight Systems, Lilienthalplatz 7, 38108 Braunschweig, Germany (until October 2013). Researcher/flight test engineer, Aerospace Technology and Science Department (DCTA) - Flight Test and Research Institute, Praça Mal. Eduardo Gomes 50, 12228-901 São José dos Campos, Brazil (since November 2013).

${ }^{\dagger}$ Leader of the Cognetics Group, Institute of Flight Systems, Lilienthalplatz 7, 38108 Braunschweig, Germany.
} 
The use of passive sidesticks has been a recurrent topic of discussion since its introduction in civil aviation. The passive sidesticks associated with the FBW technology eliminated some desirable characteristics of the inceptors used in conventional flight control system aircraft. Maybe the most important feature lost was the coupling between both sidesticks. This means that the pilot monitoring (PM) cannot assess the control inputs of the pilot flying (PF). This problem has been referred to as the break of a communication link between pilots. Another problem that can arise from uncoupled sidesticks is a dual input situation. This happens when both pilots actuate simultaneously on their sidesticks. Both problems have been related to unfortunate events, ${ }^{2-6}$ as discussed in detail by Uehara. ${ }^{7}$

In the case of the Airbus A320, the choice not to have a mechanical coupling was to avoid the introduction of single failures that could affect both sidesticks, thereby requiring a separation system, and also to avoid the introduction of friction, backlash and inertia. ${ }^{8}$

The development of active inceptor technology has made it possible to electronically couple two sidesticks. ${ }^{9}$ This technology has been successfully employed in military aircraft. The Institute of Flight Systems at the DLR already has experience in using active sidesticks for pilot assistance in helicopters. ${ }^{10}$ Safety considerations in the design of active inceptor systems are given by Taylor et al. ${ }^{11}$

This research investigates the potential improvement of the PM's awareness provided by coupled sidesticks, when the airplane is manually controlled by the PF. The scenario considered for this study is the final approach and landing in manual control. The scenario and the experiment preparation are described and the results of pilot evaluations are presented.

\section{A. The Importance of the Monitoring Duties}

The Advisory Circular 120-71A, ${ }^{12}$ issued by the Federal Aviation Administration (FAA), introduced the change of designation of the "Pilot Not Flying" (PNF) to "Pilot Monitoring" in 2003. The reason for this change was to better characterize the pilots by what they are doing rather than by what they are not doing. ${ }^{12}$ Moreover, this Advisory Circular recommends to the operators to review the existing standard operating procedures (SOP) to ensure that monitoring is emphasized. It additionally suggests that high-level SOP explicitly state monitoring as a primary responsibility of each crewmember. An example is provided by the document:

\section{"Monitoring Responsibility}

- The PF will monitor/control the aircraft, regardless of the level of automation employed.

- The PM will monitor the aircraft and actions of the PF."

The Advisory Circular 120-51E $\mathrm{E}^{13}$ cites crew monitoring and crosschecking as evolving concepts of crew resource management. The document highlights that effective monitoring and crosschecking can be the last line of defense to prevent an accident because detecting an error or unsafe situation may break the chain of events leading to an accident. It also states that:

"To ensure the highest levels of safety, each pilot must carefully monitor the flight path and systems of the aircraft and actively crosscheck the actions of the other pilot."

It must be highlighted that effective monitoring and crosschecking are not only a function of the pilots' training and the pilots' attitude within the cockpit. It is also a function of the human-machine interface design of the cockpit. This study aims to demonstrate that the coupling between sidesticks is a humanmachine interface feature which enhances the resources available for the PM to monitor and crosscheck the actions of the PF.

\section{B. Previous Research}

A detailed description of previous studies related to this research is given by Uehara and Niedermeier. ${ }^{14} \mathrm{~A}$ brief description of these studies is presented in this paper for the sake of completeness.

Summers et al. ${ }^{15}$ presented a thorough investigation of sidestick inceptor characteristics in a fixed-base simulator. Among other things, they demonstrated that the response time of the PF to the inputs made by the PM were lowest with the sticks-coupled configuration for the collision avoidance and the go-around 
scenarios. The pilots preferred coupled sidesticks because they could obtain force feedback through the control stick and the stick force communicated a sense of urgency.

Field and Harris ${ }^{16}$ conducted a survey to assess how the pilots from airlines consider the utility of the communication lines within the cockpit. They have considered four communication lines: proprioceptive, central visual, aural and peripheral visual. The results have shown that the position of the control columns of the conventional flight control system (FCS) airplanes provides useful anticipatory cues of the airplane's pitch and roll motion. The results strongly suggest that the cues available to the pilots operating FBW airplanes are reduced, when compared to the conventional technology airplanes.

Rees and Harris ${ }^{17}$ made an experiment to evaluate the effect of removing the coupling between the inceptors of the instructor pilot and the trainee pilot. Their experiment results suggest that the physical linkage of the flight controls is an important channel of communication between instructor and trainee, which enhances skill development in trainee pilots.

Uehara and Niedermeier ${ }^{14}$ considered a scenario of stall in cruise flight, which was based on an accident that happened in 2009, to study the benefits of coupled sidesticks to the PM. This research was based on a simulator experiment, in which the emulation of coupled sidesticks allowed the PM to follow the movement of the sidestick by holding it. This research demonstrated that the PM can improve his/her situation awareness by assessing the PF's control inputs on the sidestick.

\section{Scenario Preparation}

\section{A. Scenario Choice}

The approach and landing phases were chosen for the scenario of this study. These flight phases impose high workload on the cockpit crew, especially in highly dense terminal areas. Additionally, a very coordinated interaction between pilots is necessary to guarantee flight safety during approach and landing.

A few flight incidents during landing $2,5,6,18$ have been related to problems of dual inputs and/or lack of the PM's perception of the PF's control inputs on the sidestick. All these events involved FBW commercial airplanes featuring uncoupled sidesticks. Additionally, these reported incidents provide factual support that highlights the relevance of this study for the approach and landing scenario.

\section{B. The Replayed Simulation Methodology}

The replayed simulation methodology consists basically of dividing a simulator experiment in two phases: "data recording phase," in which the PF performs flight tasks and the simulation data are recorded, and the "replaying phase," in which the recorded data are sent to the simulation model, so the evaluation pilot can act as the PM and take the appropriate actions. Figure 1 illustrates the implementation of the simulation model adaptations necessary for each of these two phases. The choice for the replayed simulation methodology was necessary because the generic cockpit simulator used in this study was equipped with only one active sidestick. The coupling of the active sidestick at the pilot's station to the passive sidestick at the copilot's station was considered, but due to technical restrictions the resulting delay between an input on the passive sidestick and the tracking movement of the active sidestick was too large. Thus, the actual coupling between the pilot's and copilot's sidesticks was not possible. Therefore, it was not possible to have the actual PF and PM simultaneously within the cockpit for the coupled sidesticks evaluation.

This methodology has the advantage of guaranteeing that the set of landings and all their associated characteristics are exactly the same for each evaluation pilot. This is supposed to bring homogeneity to the data gathered.

Two modified simulation models were designed to replay the recorded data. One model was implemented to emulate the sidesticks-uncoupled condition, and the other model was implemented to emulate the sidesticks-coupled condition. In both cases, at any moment during the replay, the control could be taken over by the PM (evaluation pilot).

The control takeover during replay could be done either by pressing the priority pushbutton or by applying an input to the sidestick. Both cases were considered, because it was not possible to anticipate what would be the reactions of the evaluation pilots during the experiment. In case of the control takeover by pressing the priority pushbutton, the transition between the replayed simulation and the piloted simulation was instantaneous. In case of the control takeover by an overriding input on the sidestick, the transition from the replayed simulation to the piloted simulation was not instantaneous, following specific logics designed 


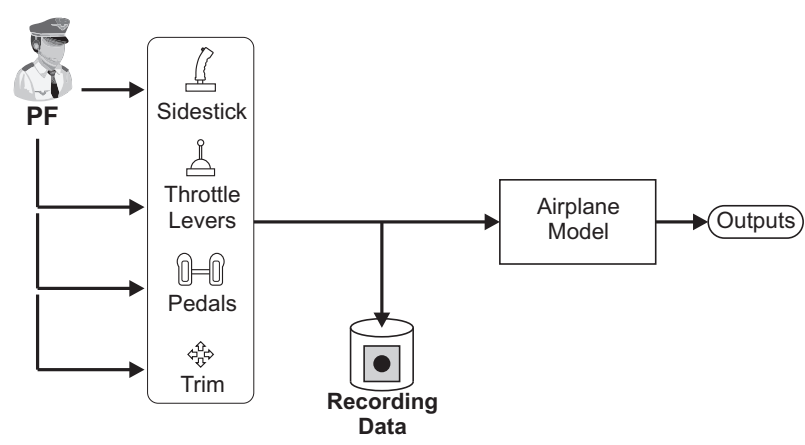

(a) Data recording phase: the PF performs landings and the simulation data are recorded.

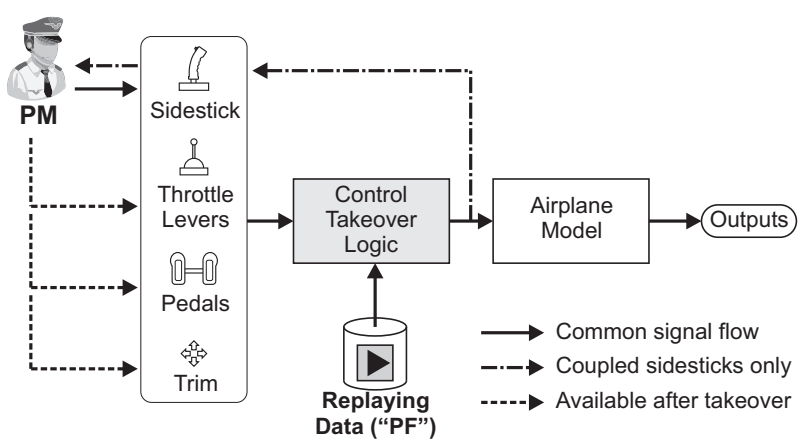

(b) Replaying phase: recorded landings are replayed for the PM. The sidestick position feedback is present only for the coupled configuration.

Figure 1. Replayed simulation methodology.

for each condition (coupled or uncoupled). The logic implemented and the hypotheses assumed for each case are described in detail by Uehara. ${ }^{7}$

\section{Gathering Landing Data}

Several approaches and landings were performed by a test pilot in the generic cockpit simulator at the Institute of Flight Systems of the DLR. All the simulation data were recorded. Three types of landings were performed as part of the experiment preparation: normal, hard, and high pitch attitude landings. The hard landings were characterized by an insufficient or absent pitch up input during flare. The high pitch attitude landings were characterized by an over commanded pitch up input during flare. In this case, the control inputs to make the nose high were commanded below $50 \mathrm{ft}$ AGL. This requirement was necessary because during the experiment the PM would be asked to take over control, if necessary, only at $50 \mathrm{ft}$ AGL or below. An illustration of the landing vertical profile is presented in Fig. 2 with additional information.

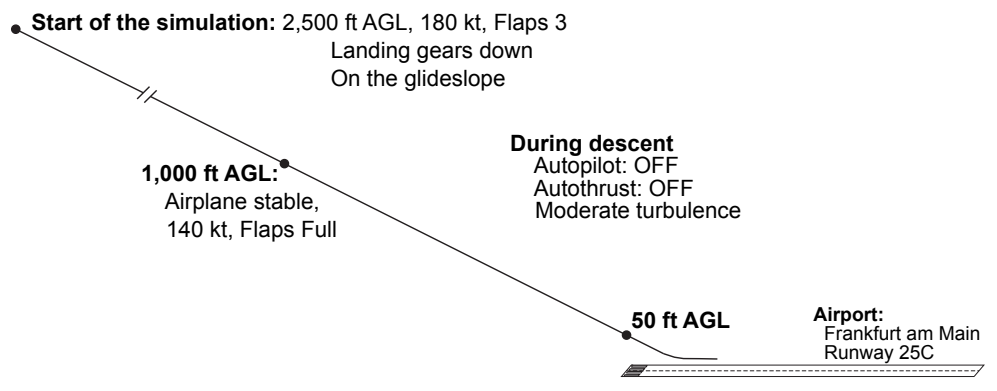

Figure 2. Landing vertical profile.

From the recorded landings, five were chosen for the experiment: one normal, two hard, and two high pitch attitude landings. Additionally, two extra recorded landings, one hard and one high pitch attitude landing, were chosen to be demonstrated to the evaluation pilots during a familiarization session prior to the experiment.

\section{Experimental Setup}

\section{A. Simulation Environment}

The experiments were conducted in a fixed-base simulator featuring dynamic model, flight control laws, automation capabilities, and a human-machine interface similar to an A320. A picture of the simulator cockpit is presented in Fig. 3.

The visual system of the simulator consisted of two projectors and a cylindrical screen, that provided a useful field of view of approximately $160^{\circ}$ horizontally and $48.6^{\circ}$ vertically from the design eye position. 


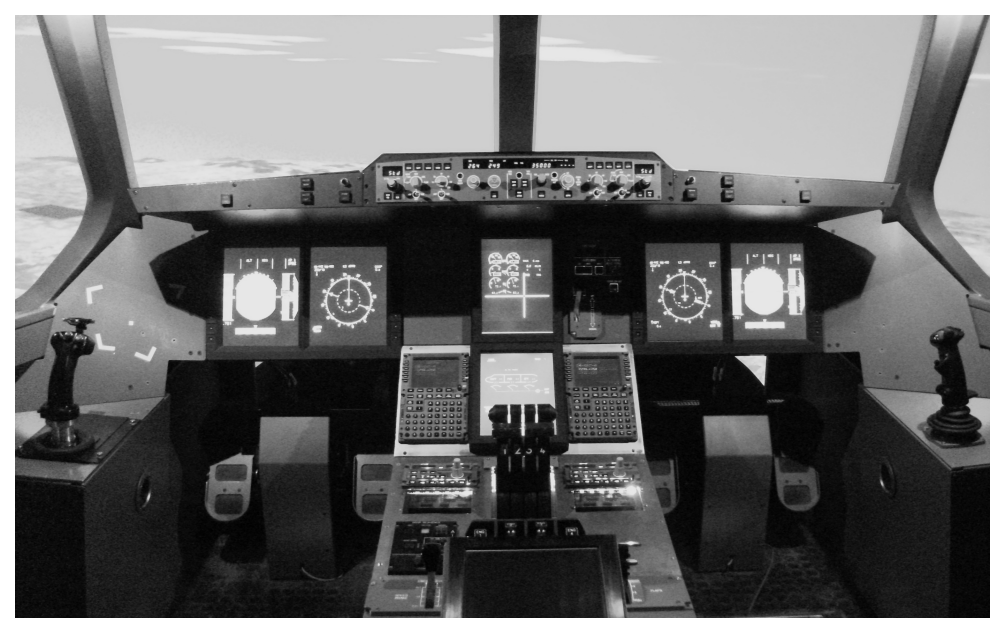

Figure 3. Generic cockpit simulator.

The simulator was equipped with one active sidestick manufactured by Wittenstein, which was installed at the captain's station. The first officer's station was equipped with a general purpose passive sidestick.

\section{B. Subjects and Briefing}

Twelve pilots participated in this evaluation. All the pilots were either experienced airline pilots or test pilots with airline experience. This is an important aspect of this research that contributes to make it representative of the real airline operation. Table 1 presents information about the pilots and their flight experience.

Table 1. Experience of the pilots.

\begin{tabular}{cccl}
\hline Pilot \# & $\begin{array}{c}\text { Total } \\
\text { Flight } \\
\text { Experience }\end{array}$ & $\begin{array}{c}\text { A320 Family } \\
\text { Flight } \\
\text { Experience }\end{array}$ & Position \\
\hline 1 & $5,950 \mathrm{~h}$ & $1,000 \mathrm{~h}$ & Test pilot (airline experience) \\
2 & $7,800 \mathrm{~h}$ & $600 \mathrm{~h}$ & Test pilot (airline experience) \\
3 & $2,500 \mathrm{~h}$ & $2,000 \mathrm{~h}$ & First Officer \\
4 & $2,000 \mathrm{~h}$ & $1,500 \mathrm{~h}$ & First Officer \\
5 & $9,060 \mathrm{~h}$ & $5,500 \mathrm{~h}$ & Captain (Airbus instructor) \\
6 & $3,600 \mathrm{~h}$ & $2,200 \mathrm{~h}$ & First Officer \\
7 & $13,970 \mathrm{~h}$ & $5,200 \mathrm{~h}$ & Captain (Airbus instructor) \\
8 & $25,000 \mathrm{~h}$ & $18,000 \mathrm{~h}$ & Captain (Airbus instructor) \\
9 & $1,600 \mathrm{~h}$ & $500 \mathrm{~h}$ & First Officer \\
10 & $4,000 \mathrm{~h}$ & $700 \mathrm{~h}$ & First Officer \\
11 & $2,000 \mathrm{~h}$ & $1,800 \mathrm{~h}$ & First Officer \\
12 & $11,400 \mathrm{~h}$ & $6,000 \mathrm{~h}$ & Captain \\
\hline
\end{tabular}

The briefing was read to the pilots prior to the simulation. They were informed about the general objective of the experiment. The pilots were informed that they would be monitoring replayed landings that had been previously recorded. They were explained that there was no real coupling of sidesticks, since there was only one active sidestick. They were told that it was possible to take over control either by applying force to the sidestick or by pressing the priority pushbutton. They were informed that the simulation model was representative of the direct law of the A320. ${ }^{19}$ They were explained that they should keep their left hand or finger tips loosely holding the sidestick during the final approach and landing. They were explained that they should take over control to correct inappropriate inputs during flare only below $50 \mathrm{ft}$ AGL. They 
were informed that the throttle and pedal commands would be available after the control takeover. Finally, they were told not to apply a force greater than half a kilogram-force on the sidestick, while holding the sidestick to follow the PF's inputs.

\section{Experimental Procedure}

The simulation session with each evaluation pilot was divided into two parts: a "familiarization session" lasting for approximately one hour, and the "experimental session" lasting for approximately one and a half hours. A short coffee break or a lunch break was made between sessions, but both sessions were always conducted within the same day.

\section{Familiarization Session}

During the familiarization session the evaluation pilots could adjust the seat, armrest, and pedals. Afterwards, they performed several approaches and landings. Initially, visual meteorological conditions (VMC) were set and no wind/turbulence was added to the scenario, but the environmental conditions were made worse as the pilots were getting more familiarized with the simulator. The final environmental conditions were IMC down to $500 \mathrm{ft}$ AGL, moderate turbulence intensity and $10 \mathrm{kt}$ wind (tail or lateral). In these approaches and landings the evaluation pilots were the PF during the whole time. The initial simulation conditions are presented in table 2

Table 2. Simulation Initial Conditions

\begin{tabular}{ll}
\hline \hline - $180 \mathrm{KCAS}$ & - Flaps: 3 \\
- $2,500 \mathrm{ft}$ AGL & - Landing gears: down \\
- Autopilot: disengaged & - Ground spoilers: armed \\
- Autothrust: disengaged & - Airport: Frankfurt am Main \\
- Flight director: off & - Aligned with runway 25C \\
- Direct law & - On the glide path \\
\hline \hline
\end{tabular}

After the approaches and landings as PF, two examples of the experimental situation were demonstrated to the evaluation pilots. They could feel the sidestick movement emulating the coupling between the PF and PM sidesticks. They could act as the PM and take over control. At the end of the familiarization session the formal briefing for the experimental session was given.

\section{Experimental Session}

The experimental session consisted of ten approaches and landings. The five recorded landings chosen for this session were replayed twice for each evaluation pilot. Once with uncoupled sidesticks and once with coupled sidesticks emulation. The sequence of the replays was random, but it always alternated between one uncoupled and one coupled. Additionally, the same landing featuring different coupling configurations was never presented twice in a row. The evaluation pilots acted always as the PM and they occupied the commander's station. The first officer's station was occupied by an engineer, who interacted with the evaluation pilot by making the applicable callouts during the approach phase. The engineer held the sidestick and the throttle levers, as it would have been done in case a real PF was present. The initial conditions of the simulation were the same as those from the familiarization session presented in table 2. Following each landing the simulation data files were saved for later analysis. After the ten landings were performed the evaluation pilots were requested to answer to a questionnaire and they were interviewed.

\section{Scenario Limitations}

Some technical restrictions imposed the following limitations on the experiment scenario:

- The throttle levers were not active, so they could not move during the replay to reproduce the inputs applied by the PF.

- The pedals could not move to emulate the PF's inputs for the same reason as the throttle levers. 
- Since there was no real coupling between the sidesticks, there was no real PF, so interactions between the crewmembers were limited.

- There was no ATC communications. It was considered reasonable to assume that clearance to land had been granted. Nevertheless, the presence of ATC communications, even with other aircraft, would provide extra workload and improve the evaluation pilot's immersion.

- The simulator had no motion system. Although some studies do not seem to support an operational benefit of motion systems of full flight simulators for transfer or quasi-transfer of training, ${ }^{20}$ the effect of the absence of motion cues to the PM is not clear. Nevertheless, those cues are expected to have secondary importance when compared to visual cues.

These limitations were considered of secondary importance. The experiment setup was considered realistic enough and representative of the real airline operation. The scenario was reviewed at the simulator by a test pilot who was type rated in the A320. The questionnaire was reviewed by a member of the Cognetics Group of the Institute of Flight Systems at DLR.

\section{Results and Discussion}

The results presented herein provide the most important findings of this study and the most relevant opinions of the pilots who have participated in the simulator experiment. For more details, the reader can refer to Uehara. ${ }^{7}$

The three-level model of situation awareness (SA) defined by Endsley, ${ }^{21}$ which is an information processing model, was considered to evaluate the sidestick movement as a cue to the PM. The levels of SA as defined in this model are perception, comprehension, and projection, respectively levels 1,2 and 3. The definition of this model states that situation awareness is:

"The perception of the elements in the environment within a volume of time and space, the comprehension of their meaning, and the projection of their status in the near future."

\section{A. Answers to the Questionnaire}

\section{Subjective Assessment of the Sidestick Cues for Situation Awareness and Decision Making}

In this part of the subjective evaluation, the pilots should rate the usefulness of the sidestick movement for perception and projection, respectively levels 1 and 3 of SA. Additionally, they should rate the usefulness of the sidestick movement for decision making when a control takeover was necessary.

The first question assessed the perception (SA level 1) that the evaluation pilots had of the PF's inputs (Fig. 4a). All 12 evaluation pilots considered the coupling between sidesticks at least as "useful" to improve their perception of the PF's inputs. Nine pilots rated the coupling "very useful" or "extremely useful", as shown in Fig. 4a. This result suggests that the coupling was effective to convey the information about the PF's control inputs to the PM. As the first level of SA, perception of relevant information is the base for the pilots to build the correct picture of the situation. A study made by Jones and Endsley ${ }^{22}$ showed that the great majority of the SA errors in aviation are related to problems in the perception level. Moreover, it is reasonable to assume that once the PM can perceive the PF's inputs, his/her awareness is improved, at least concerning the manual control of the airplane. The possibility for a pilot to perceive the other pilot's control inputs on the sidestick enables redundancy of judgment of the suitability of these inputs. Additionally, some unfortunate events have been related to inappropriate inputs on the sidesticks. ${ }^{2,3,5,6,18,23}$ It is likely that the coupling between sidesticks can decrease the odds of such events.

The second question assessed the anticipation of the airplane behavior by the PM, when he/she followed the sidestick movement in the coupled configuration (Fig. 4b). The anticipation is expected to exist because in large transport FBW airplanes the response to a sidestick input comes with a delay. The response delay is basically due to the processing of the inputs by the flight control computers, but it can also include a delay due to actuator rate limit. In terms of situation awareness theory, this anticipation was treated as a projection of the airplane's status in the near future, therefore related to the third level of situation awareness in Endsley's model. ${ }^{21}$ The answer distribution of the second question is presented in Fig. 4b. It shows that 11 pilots considered the sidestick movement at least useful to anticipate the airplane behavior due to the 


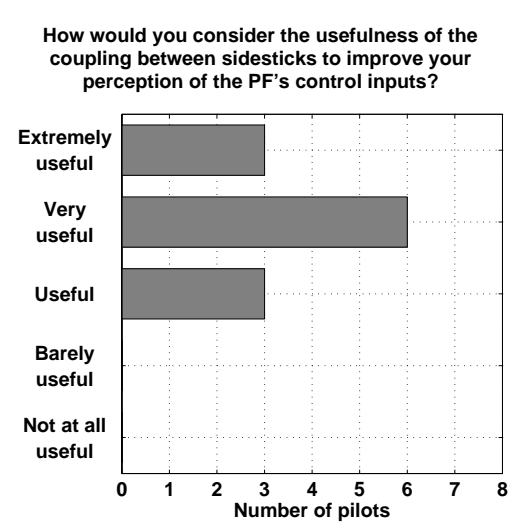

(a) Question \#1: Assessing coupling of sidesticks for perception (SA level 1).

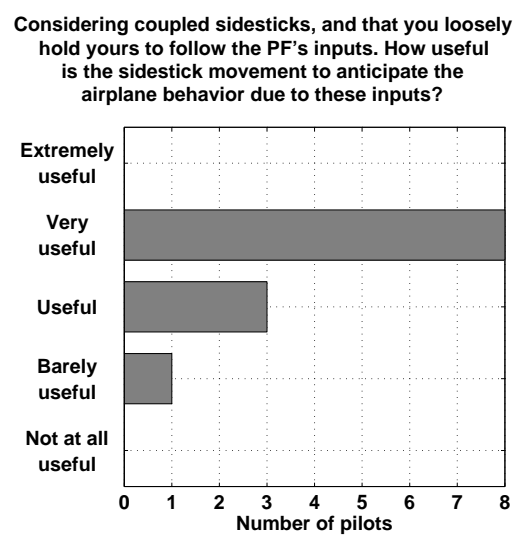

(b) Question \#2: Assessing coupling of sidesticks for projection (SA level 3).

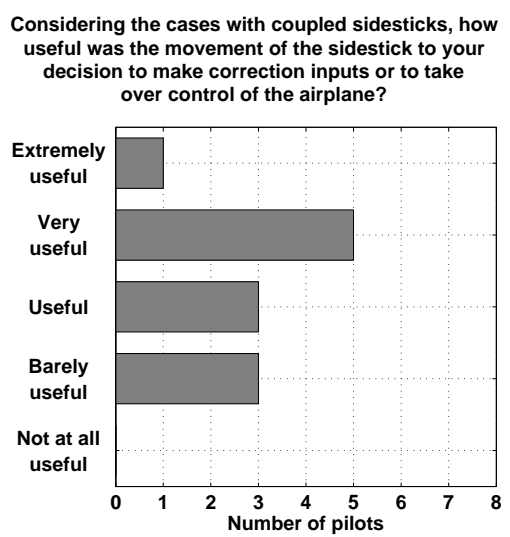

(c) Question \#3: Assessing coupling of sidesticks for decision making.

Figure 4. Answer distributions for the subjective evaluation.

PF's control inputs. This is a relevant result which suggests that the anticipation, although not large, can indeed be achieved by the pilots. A discussion about whether this anticipation influences the PM's decision for a control takeover is presented afterwards.

The third question assessed the usefulness of the sidestick movement as a contribution to the PM's decision to make correction inputs or to take over control of the airplane in case of inappropriately executed flares (Fig. 4c). The answer distribution presented in Fig. 4c shows a relatively higher dispersion of opinions compared to the previous two questions. Situation awareness is a major input for decision making. ${ }^{24}$ If the PM's awareness concerning the airplane control is improved by coupled sidesticks, then the sidestick movement should provide a better basis for the PM's decision to take over control. Indeed, the answer distribution in Fig. 4c shows that 9 pilots considered the sidestick movement at least "useful" for their decision to take over control. Additionally, no pilot said that it was "not at all useful". The reason for the dispersion of opinions is probably related to the fact that operationally the decision to take over control relies on the observation of other parameters that are displayed on the PFD, for example the pitch attitude and airspeed. Prior to decide for a takeover, the PM makes a callout informing the PF about the parameter that has gone beyond the operational limits, as described in the flight manual. ${ }^{25}$ The control takeover happens only if no appropriate corrective action is taken by the PF. Moreover, it is worth noting that operationally a control takeover during flare will always lead to a go-around.

\section{Comparison Between Coupled and Uncoupled Sidesticks for Situation Awareness and Workload}

In this part of the subjective evaluation, the pilots should compare coupled/uncoupled sidesticks for perception and projection, respectively levels 1 and 3 of SA. Additionally, they should compare coupled/uncoupled sidesticks for the workload to monitor the PF.

The fourth question (Fig. 5a) compared the perception that the PM had of inappropriate control inputs made by the PF during flare with coupled and uncoupled sidesticks. The answer distribution is shown in Fig. 5a. It suggests that the PM had a better perception of inappropriate control inputs of the PF during flare with coupled sidesticks. With uncoupled sidesticks, an unsuitable control input during flare would be ultimately identified by the PM indirectly through the observation of the inadequate outputs, namely pitch rate, pitch angle, descent rate, roll rate, bank angle, deviation from the runway, etc. As explained in the discussion of the third question, operationally these output parameters are monitored and used by the PM for the decision of a control takeover, if no corrective action is taken by the PF. It is interesting to crosscheck the answers to this question with the answers to question \#1. In that question, the sidestick movement was considered on average "very useful" to improve perception of the PF's inputs. Thus, it would be expected that an inappropriate input could be perceived more easily with coupled sidesticks. Indeed, the answer distribution in Fig. 5a provides the confirmation.

The fifth question (Fig. 5b) compared the anticipation of the airplane dynamic behavior provided by coupled and uncoupled sidesticks. The answer distribution shown in Fig. $5 \mathrm{~b}$ seems to be consistent. As explained in the discussion of the second question, the anticipation is possible due to the delay between a 


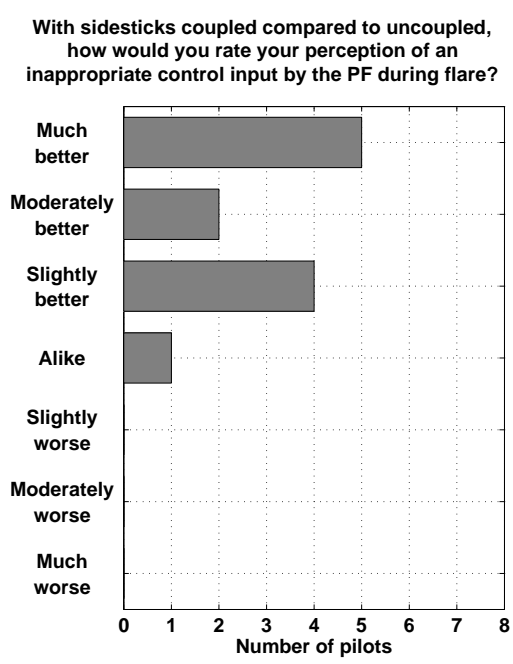

(a) Question \#4: Comparing perception of inappropriate inputs. (SA level 1)
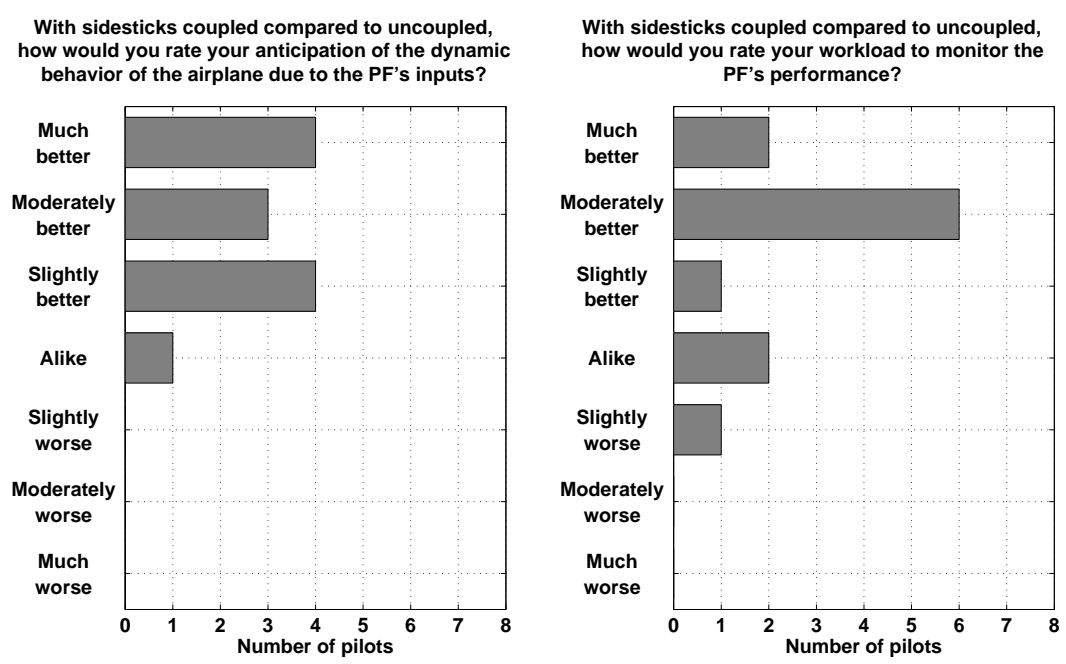

(b) Question \#5: Comparing anticipa- (c) Question \#6: Comparing workload to tion of the aircraft dynamics. (SA level monitor PF.

3)

Figure 5. Answer distributions for the comparative evaluation.

sidestick input and the airplane response. The uncoupled-sidesticks configuration provides no direct feedback to the PM about the PF's inputs on the sidestick. Thus, any anticipation of the aircraft behavior would be provided by the beginning of a motion. A comparison of the answers to this question with those to question \#2 shows consistency. In that question the sidestick movement was considered, by 11 pilots, at least as "useful" to anticipate the dynamic behavior of the airplane. Thus, it would be expected that the anticipation would be better in the coupled configuration, as indeed observed in Fig. 5b.

The sixth question (Fig. 5c) is a subjective assessment of the influence of coupled sidesticks on the PM's workload to monitor the PF. The answer distribution in Fig. $5 \mathrm{c}$ shows that 8 pilots considered the workload with the coupled configuration at least "moderately better." This result suggests that the sidestick movement provided relevant information that required low cognitive effort from the PM. During debriefing of the experiment one of the pilots gave an example of how the workload is decreased. He explained that the sidestick cues conveyed the information about corrective inputs by the PF. Thus, following a glideslope or localizer deviation, the PM could compare the PF's inputs with the deviation direction. From this comparison, the PM would readily become aware of whether the deviation was being corrected or not. In case of corrective inputs by the PF, the PM could be alleviated from the task of further monitoring the deviation, otherwise he/she would make a callout when the deviation reached the operational limit of $1 / 4$ dot of localizer and 1 dot of glideslope. ${ }^{25}$

\section{B. Interview}

After completing the questionnaire the pilots were interviewed. The interview consisted of seven questions that are presented below. The questions are followed by the most relevant answers from the pilots and by a discussion of these answers.

\section{Question 1, answers and discussion}

In the coupled sidesticks configuration, would you consider the movement of the sidestick annoying or distracting?

Most of the pilots considered the sidestick movement neither annoying nor distracting. This is an important result which suggests that coupled sidesticks may be well accepted by the pilots. Other relevant comments:

- Pilot 5 explained that the PM uses the press-to-talk button (trigger switch) of the sidestick to communicate with the ATC, and it could be annoying if the sidestick were moving. 
- Pilot 7 answered that during the first landing with coupled sidesticks it was annoying. After two or three approaches, he adapted himself and it was getting better.

The problem raised by pilot 5 could be solved during the design phase of the cockpit layout by placing the press-to-talk switch elsewhere. It should be remembered that this research is aimed at future generations of commercial FBW airplanes, and by no means it is suggesting changes to the currently flying airplanes.

The comment made by pilot 7 suggests that the adaptation to the moving sidestick is easy and quick. This affirmation is also supported by the fact that the other pilots who considered the sidestick movement neither annoying nor distracting made no comment regarding difficulty to adapt themselves.

\section{Question 2, answers and discussion}

If somehow the moving sidestick constituted a source of distraction or bother, it could degrade the overall awareness of the PM by deviating his/her attention from other relevant sources of information. To assess whether this was the case or not, the following question was raised:

Do you believe that to follow the PF's inputs by holding the sidestick had a negative impact on your overall situation awareness?

Eight pilots were convinced that coupled sidesticks had a positive impact to their overall SA. One pilot answered that the coupling had neither positive nor negative impact. Further relevant comments provided by the pilots are listed below:

- Pilot 2 was among the 8 pilots who were convinced about the positive impact of coupled sidesticks to the SA. He said that, sometimes in real operation, he looks to the PF's sidestick to assess his/her control inputs. He explained that with uncoupled sidesticks it is not possible to know whether some movements of the airplane are due to wind/turbulence or due to PF's control inputs.

- Pilot 5 said that coupled sidesticks would be useful to improve situation awareness in very limited cases. He explained that the outside view constitutes more direct information during flare. Additionally, he explained that the informations on the PFD are objective, whereas the sidestick movement is subjective.

- Pilot 7 explained that to use the sidestick cues effectively training is necessary. A pilot would need to learn and to build a background in using these cues. He recognized that the sidestick provides some cues. "The cues are there." He explained that due to his experience as an instructor in the A320, he is used to assess the PF's workload by subjective cues. Among these cues he cited the inputs that the $\mathrm{PF}$ applies to his/her sidestick, sweat and eye movement. He said to be trained to compensate the uncoupled sidesticks.

- Pilot 12 said that pilots are used to fly with uncoupled sidesticks, and that the effect of wrong inputs can be seen in the ADI. He explained that training would be necessary to make the use of the sidestick cues more effective.

The comment of pilot 5 does not imply any negative impact of coupled sidesticks on the situation awareness. Indeed, he affirmed that it would improve awareness, although in very limited cases.

Pilot 7 provided important comments about the need of training and building a background to effectively use the sidestick cues. His comment concerning the compensation of uncoupled sidesticks suggests that it is possible for an instructor to live without the coupling, but it also sounds like a useful feature is missing in the pilot-airplane interface.

The comments of pilot 12 might suggest that the sidestick cues are unnecessary given the fact that pilots are used to fly with uncoupled sidesticks and the possibility to observe wrong inputs in the ADI. However, none of these extra comments provided by the pilots to this question suggested that to follow the PF's inputs could degrade the PM's overall situation awareness. This result suggests that, concerning specifically the situation awareness issue, there would be no drawback by coupling the sidesticks. 


\section{Question 3, answers and discussion}

This experiment considered only the final approach and landing phases. To identify other flight phases or conditions in which the coupling between sidesticks would be relevant for the PM, the following question was made:

Can you describe other situations in which coupled sidesticks would be beneficial?

The following list presents a compilation of the answers obtained from this question:

- During takeoff rotation (pilots $2,3,5,6,7,9$ and 12 ). In this case it would be possible to identify early rotations (pilot 7). It would be possible to feel how young pilots rotate during training (pilot 5).

- During crosswind, wind gusts and turbulence situations (pilots 1, 4, 8, 10 and 12). The perception of the PF's control inputs would be much more direct (pilot 10).

- In special situations that require special landing techniques as, for example, extreme center-of-gravity loadings (pilot 4).

- In cruise when the autopilot disengages due to strong turbulence to assess the PF's reaction (pilot 9).

- During a wind shear escape maneuver (pilots 2 and 6 ) or during a ground proximity warning (pilot 3). The procedure in both cases is to apply TOGA power and full aft sidestick deflection. In these maneuvers it is relevant to know what the PF's inputs are.

- In case of an unreliable airspeed event (pilot 12) or during stall in cruise, when a nose down input is expected (pilot 2). In this case, both pilots referred to an accident over the Atlantic Ocean in 2009. ${ }^{23}$

- The sidestick movement could provide feedback in all dynamic flight conditions (pilot 7).

- Always when manually flying in IMC conditions (pilot 12).

It seems that the sidestick movement cues are considered more important during landing, as highlighted by pilots 5 and 8 . Nevertheless, the list of situations compiled from the interview with the pilots suggests that the sidestick cues may be relevant also in other scenarios besides those considered in this research. It is recommended that further experiments be conducted in scenarios different from those considered in this research to further assess the benefits of coupled sidesticks.

\section{Question 4, answers and discussion}

In order to obtain the evaluation pilots' opinion about the relevance for the PM to know the PF's control inputs, the following question was made:

\section{In your opinion, how relevant is it for the PM to know the control inputs being made by the PF?}

In this question, the pilots could choose one answer among four options and they could make comments. The results are presented in Fig. 6.

Pilot 2 explained that to know the PF's control inputs is "moderately relevant" during takeoff, landing and recovery maneuvers from unusual situations in any flight phase. In normal operation, during climb, cruise and descent the airplane is flown by the automatic flight systems, so there is no PF's control inputs. Nevertheless, if for any reason the airplane were manually controlled by the PF during these flight phases, to know the PF's inputs would be "not relevant at all". The answer considered from pilot 2 in the results was "moderately relevant" because the question was considering the experiment scenario.

Pilot 7 answered "moderately relevant" to this question, but he emphasized that more relevant than the inputs are the results of the inputs. In his words, "the feedback alone does not tell everything". Pilot 8 answered "very relevant" and 
specified during flare. Pilot 10 answered "very relevant" during takeoff and landing. Additionally, he said that the more adverse the weather, the more interesting it is to have the cues of the PF's control inputs.

Nine pilots considered to know the PF's control inputs at least "moderately relevant", for the scenario of this experiment. It should be remembered that the evaluation pilots were used to uncoupled sidesticks. It is likely that training and experience with coupled sidesticks would improve the effectiveness of use of the sidestick movement as a cue, and probably make the information more relevant for the pilots.

\section{Question 5, answers and discussion}

In order to assess how desirable it is to have coupled sidesticks, the following question was made to the evaluation pilots:

\section{How desirable would you consider the coupling between sidesticks in the cockpit?}

In this question, the pilots could choose one answer among four options and they could make comments. The results are presented in Fig. 7.

Reflecting his comment on question 4, Pilot 2 explained that during takeoff, landing and recovery maneuvers from unusual situations the coupling between sidesticks is considered "highly desirable". In normal operation during climb, cruise and descent it is considered "indifferent".

Pilot 7 answered "indifferent", but he added that if he had to take a decision about coupling the sidesticks or not, he would decide for coupling. Nine pilots considered the coupling between sidesticks at least "desirable". Since it was already seen that coupled sidesticks were considered useful and provided relevant information for the PM, this result seems consistent. It is important to note in Fig. 7 that no pilot considered the coupling undesirable, which suggests that the

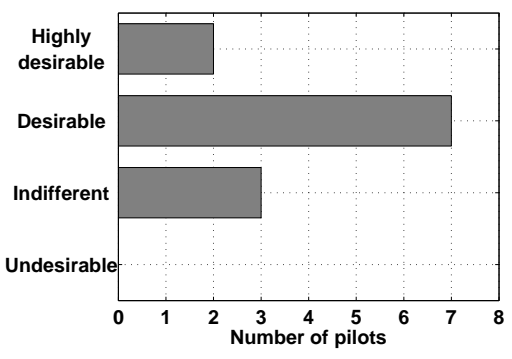

Figure 7. Answer distribution of the fifth question of the interview.

\section{Question 6, answers and discussion}

An open question was made at the end of the interview to allow the gathering of unanticipated information that could be valuable to this research:

Do you have any extra comment pro or against the coupling between sidesticks?

The most relevant comments of the pilots were listed below for further discussion. For the complete list the reader should refer to Uehara. ${ }^{7}$

- Pilot 2 explained that it was easier to perceive inputs that led to high pitch attitude flares, than to perceive inputs that led to hard landings. In his opinion, for the hard landing case, the sidestick movement did not make much difference.

- Pilot 4 explained that it is difficult to take the decision to take over control based on the sidestick movement. Parameters such as airspeed, pitch angle, altitude, etc, presented in the PFD are objective information that "do not lie". They are measured parameters. The sidestick movement is subjective.

- Pilot 5 explained that the sidestick cues might raise a problem of information management. He said, that he prioritized the observation of the indications on the PFD and the outside view references. Then, he interpreted the sidestick movement. In his opinion, the indications and the outside view are more important. Moreover, when approaching the ground it is more difficult to manage the information available. He also suggested that time to gain experience is necessary for pilots to learn to interpret the sidestick movement.

- Pilot 7 explained that, with uncoupled sidesticks, when the priority pushbutton is pressed there is an abrupt transition of the control input. The overriding pilot has to try to match the input being made to avoid this abrupt transition. In case of coupled sidesticks, the sidestick is already in the actual commanded position, thus there is no abrupt transition. 
- Pilot 8 said that the coupling feature is useful especially in the training phase. He said that sometimes he looks to the trainee's right hand during the first flight hours to assess his/her control inputs. Concerning the experiment scenario, the pilot believes that the sidestick movement can anticipate the decision to take over control. He explained that with uncoupled sidesticks the captain has to wait until a deviation from the desired flight path occurs, and then he/she takes over. With coupled sidesticks the captain does not need to wait for a flight path deviation, because he/she can recognize inappropriate control inputs earlier. The pilot explained that the delay between the sidestick input and the airplane response could be eliminated by directly assessing the PF's sidestick inputs. He said to be convinced that many hard landings and go-arounds in commercial aviation could have been avoided if the sidesticks were coupled.

- Pilot 9 explained that the feedback of the PF's inputs was immediate.

- Pilot 10 explained that the decision to take over control is based on the external view and on the instrument indications. He recognized that the inputs are perceived first, followed by the airplane response and then the instrument indications. However, the pilots are used to rely on the instruments.

- Pilot 12 said that the coupling is a good feature for the instructors to feel the trainee control inputs.

An overview of the comments provided by the pilots in this open question shows a wide variation of opinions, sometimes divergent. Such heterogeneity of comments is probably related to the fact that none of the pilots had experience with coupled sidesticks. Another reason may be the differences of backgrounds.

From the comments of pilots 8 and 12, it seems that coupled sidesticks are a useful feature for the instructor pilot during the training phase of new copilots. This issue was also addressed by Rees and Harris, who analyzed the benefits of coupled sticks for the trainees. ${ }^{17}$

The problem of information management suggested by pilot 5 , if indeed relevant, might be attenuated by training and by the experience that would be achieved through the day-by-day operation with coupled sidesticks. As also implied by pilot 5 , time is necessary for the pilots to learn to use the sidestick movement as a cue. Additionally, based on the results of question 1 of this interview, it seems unlikely that the movement of the sidestick could constitute a source of disturbance for the PM. Moreover, commercial airplanes with control columns like, for example, those from Boeing and Embraer have coupled control inceptors and, for the PM, there is no record of problems caused by the movement of the column/yoke. On the contrary, the coupling is actually a feature appreciated by pilots of those airplanes.

The comments from pilots 4, 5 and 10 suggest that to decide for a control takeover based on the sidestick cues might not be appropriate. From their comments it is suggested that the indications on the PFD are more objective, and therefore more important, than the sidestick inputs when it comes to decide for a control takeover. The outside view is another important information considered. Indeed, the limits for the deviations of airspeed, sink rate, bank angle, pitch angle, localizer, glideslope, and course are defined within the standard operating procedure ${ }^{25}$ of the A320 for the approach and landing phases. Of course, to establish operational tolerances or limits for the sidestick deflection is not under consideration. Nevertheless, the PF's inputs on the sidestick ultimately influence pitch attitude, bank angle, sink rate, and flight path during flare. It is clear that the pilots rely on the instruments to make decisions, as highlighted by pilot 10. However, the sidestick cues are not supposed to substitute any instrument. Since the inputs have direct correlation with several monitored parameters, the perception of these inputs by the PM can provide him/her with a more complete comprehension of the cause-and-effect relationship behind the airplane's dynamic behavior during a manually piloted approach and landing. This is probably one explanation why the sidestick cues were considered at least "useful" for the decision to take over control by 9 pilots in the third question of the questionnaire.

The comment of pilot 8 suggests that the anticipation of the inputs with respect to the airplane response could even permit an early decision to take over control. Indeed, an anticipation of the airplane dynamics could be achieved by the pilots as it is shown by the responses to the second and fourth questions of the questionnaire. Nevertheless, this does not necessarily mean that the decision for a control takeover could be made earlier. This issue is discussed in detail in the next section.

The comment of pilot 7 suggests that the transition during a control takeover is smoother with coupled than with uncoupled sidesticks. Although a very interesting observation, the relevance of such improvement in the transition of a takeover is not clear, and this effect could not be observed in the results of this experiment. It is recommended that further investigations be conducted to assess this issue. 


\section{Analysis of the Simulation Data}

The objective of the analysis of the simulation data was to verify whether the presence of the sidestick cue had any influence on the reaction time of the evaluation pilots during inappropriately commanded flares.

Anticipation and delay of a takeover are herein defined by the difference between the time that a specific pilot took over control with uncoupled sidesticks and the time that he/she took over with coupled sidesticks in two replays of the same recorded landing:

$$
\Delta t=t_{\text {takeover }}^{\text {uncoupled }}-t_{\text {takeover }}^{\text {coupled }}
$$

$\Delta t>0$ means that the takeover was anticipated.

$\Delta t<0$ means that the takeover was delayed.

In total, there were 21 anticipated takeover events and 19 delayed takeover events. Figure 8 shows the anticipation or delay measured for each evaluation pilot in each of the four bad landings of the experiment. The distribution of the anticipated and delayed takeovers was random within each analyzed landing. In other words, there was no clear tendency of anticipation of control takeovers due to the presence of the sidestick movement cue, as can be seen from the analysis of Fig. 8.

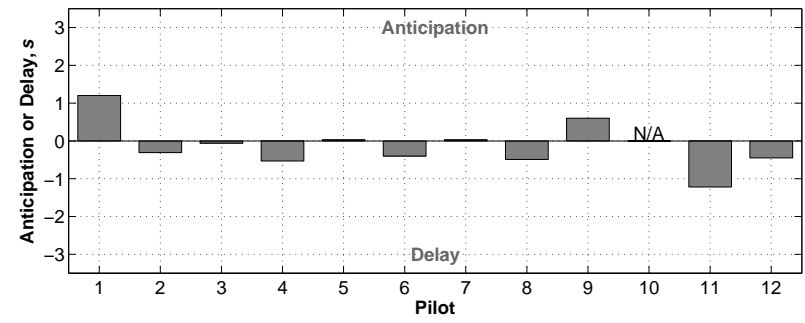

(a) Hard landing \#1.

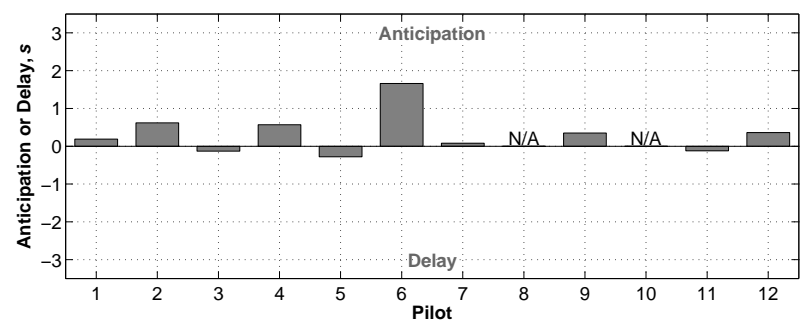

(c) Hard landing \#2.

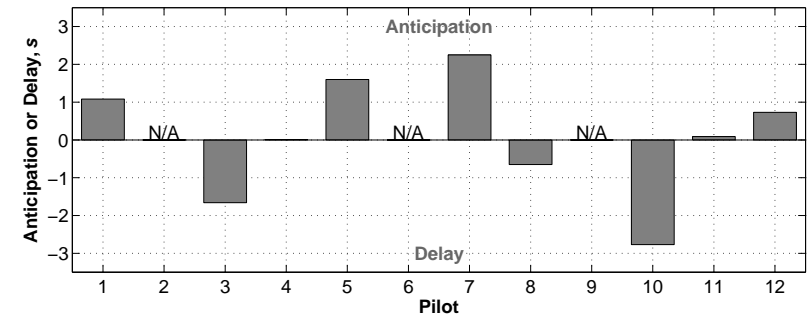

(b) High pitch landing \#1.

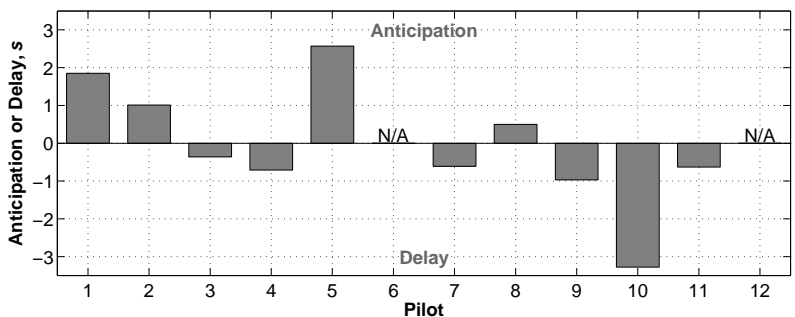

(d) High pitch landing \#2.

Figure 8. Time difference between control takeovers by the PM with coupled and uncoupled sidesticks during inappropriately commanded flares. N/A means that the data could not be calculated due to one of two reasons: either the data were not correctly recorded during the experiment or one of the two landings necessary to calculate the time difference was invalid.

To summarize the analysis of the simulation data, a clear influence of the sidestick coupling to the initiation moment of the control takeovers could not be observed. The sidestick movement, transmitting the PF's inputs to the PM, provided no anticipation of the decision for a control takeover during inappropriately commanded flares, under the experiment conditions. This happened despite the comments of pilots number 2 and 8 during the interview. Pilot 2 implied that the sidestick movement was helpful to perceive inputs that would lead to a high pitch attitude flare. Pilot 8 said that the sidestick cues could anticipate the decision to take over control. This might be true in some very specific situations, if the PM is a very experienced pilot, as it was the case of pilot 8 . The anticipation of the control takeover did not occur because the pilots used the same decision strategy with coupled and uncoupled sidesticks. They basically did what they were trained to do: follow the standard operating procedures. Concerning the high-pitch-attitude landings, in general the pilots observed the operational limit of $7.5^{\circ}$. It is likely that the experience that pilots would build by operating an airplane with coupled sidesticks would refine their skill of perceiving inadequate inputs, as implied by pilots 5,7 and 12 in the interview. The anticipation of a decision, if possible, is not expected to be large. The relevance of a slightly earlier decision to take over control even during flare, which is a very critical flight phase, is not clear. However, as discussed in question 7 of the interview, the sidestick movement 
is useful for the control takeover decision by providing an improved comprehension of the cause-and-effect relationship behind the airplane's dynamic behavior. Consequently, the decision, as a cognitive process, is made with enhanced awareness.

\section{Limitations of this Experiment}

Although the number of pilots who participated in the experiment was reasonable, it was not enough for a statistical analysis of the data. In order to obtain a confidence level assessment of the results presented in this study, the experiment should be repeated with a larger population.

The experiment consisted of several approaches and landings in which the evaluation pilots knew that sometimes the PF would make a mistake. Therefore, it was not possible to provoke the "startle" or "surprise" effect as it probably would have happened in a real flight condition. Nevertheless, this fact has not compromised the possibility of a subjective evaluation based on the comparison between the coupled and uncoupled sidesticks.

The subjective assessment of SA based on questionnaire answering after the simulation has its limitations. One of the main points of criticism of subjective assessment of SA is that the pilots cannot be aware of their own lack of SA. ${ }^{26}$ Besides, the method relies greatly on the pilot's judgment and memory.

\section{Conclusion}

A study about the influence of coupled sidesticks on the PM's awareness has been performed. A fixedbase simulator was used, which was representative of a commercial fly-by-wire airplane. The flight scenario was representative of the real operation and consisted of approaches and landings, some of which had inappropriately commanded flares. An active sidestick was installed at the commander's station and it could be configured to behave either as being coupled or uncoupled to the PF's sidestick. The evaluation pilots acted as the PM. During each landing, they had to monitor the PF and decide whether or not a control takeover was necessary during flare. In the approaches and landings with coupled sidesticks the PM could assess the manual inputs of the PF on his/her sidestick. Subjective data were gathered by applying a questionnaire and interviewing the pilots after the experiment. Data were recorded from the simulator to assess the response time of the PM.

Coupled sidesticks are recommended to improve the PM's situation awareness. The results show that the coupling is useful to improve the PM's perception of the PF's control inputs. Additionally, this perception was considered useful for the PM to anticipate the dynamic behavior of the airplane and it was also useful for the PM's decision to take over control. However, no anticipation of the decision to take over control was observed. Moreover, the PM's workload due to the task of monitoring the PF was lower with coupled sidesticks.

The sidestick movement was neither considered annoying nor distracting by the evaluation pilots. In general, pilots considered it desirable to have coupled sidesticks. It is noteworthy that most of the evaluation pilots considered it relevant to know the control inputs of the PM during approach and landing. Besides the approach and landing phases, several other situations in which coupled sidesticks could be useful were identified in the interview with the pilots.

The results found are based on the experiment with 12 pilots. In order to obtain a confidence level assessment of the results presented herein, the experiment should be repeated with a larger number of pilots, so statistics could be applied.

This study demonstrated that the coupling between sidesticks is an important feature of the humanmachine interface in the cockpit of a commercial fly-by-wire airplane which enhances the resources available for the PM to monitor and crosscheck the actions of the PF. By enabling the PM to assess the PF's control inputs, coupled sidesticks enable redundancy of judgment of the suitability of these inputs and, therefore, contributes to flight safety.

\section{Acknowledgments}

The authors would like to thank the following individuals for their important contributions to the realization of this study: Dr. Sven Oppermann, Mr. Christian Hoffmann, Dr. Nicolas Fezans, Dr. Holger Duda, Mr. Hans-Jürgen Berns, and Mr. Jens Heider. Dr. Oppermann and Mr. Hoffmann provided outstanding 
support with the generic cockpit simulator used in this study. Dr. Fezans made a thorough revision of the questionnaire and provided interesting suggestions to improve it. Dr. Duda provided valuable administrative support. Mr. Berns provided the contacts with the airline pilots who have participated in this research. Mr. Heider provided great support in the preparation and review of the scenario and questionnaire. Additionally, the first author would like to thank the second author for the great support and sharing of experience.

\section{References}

\footnotetext{
${ }^{1}$ Hegg, J. W., Smith, M. P., Yount, L., and Todd, J., "Features of Active Sidestick Controllers," IEEE AES Systems Magazine, Vol. 10, No. 7, 1995, pp. 31-34.

${ }^{2}$ Aircraft Accident Investigation Board (AAIB), "AAIB Bulletin: Incident with the Airbus A321-211 EI-CPE at the London Heathrow Airport on June 21st, 2000," United Kingdom, 2000.

${ }^{3}$ Comisión de Investigación de Accidentes e Incidentes de Aviación Civil (CIAIAC), "Report IN-029/2006: Wake turbulence encounter in high-level air space involving an Airbus A320, registration EC-JDK, operated by Vueling, in route between Barcelona and Santiago de Compostela on 28 May 2006," Comisión de Investigación de Accidentes e Incidentes de Aviación Civil (CIAIAC), Spain, 2006.

${ }^{4}$ Aircraft Accident Investigation Board (AAIB), "AAIB Bulletin No: 12/2004: Incident with the Airbus A320-232 GTTOA near Malaga, Spain, on April 15th, 2004," United Kingdom, 2004.

${ }^{5}$ Aircraft Accident Investigation Board (AAIB), "AAIB Bulletin No: 11/2004: Incident with the Airbus A320 C-GTDK at the Bristol Lulsgate Airport on June 16th, 2003," United Kingdom, 2004.

${ }^{6}$ Aircraft Accident Investigation Board (AAIB), "AAIB Bulletin No: 5/2001: Incident with the Airbus A321 D-AIRE at the London Heathrow Airport on October 9th, 2000," United Kingdom, 2001.

${ }^{7}$ Uehara, A. F., Use of Active Sidesticks in Multi-Crew Commercial Fly-by-Wire Airplanes, Ph.D. thesis, Technical University of Braunschweig - Faculty of Mechanical Engineering, Braunschweig, Germany, 2014.

${ }^{8}$ Corps, S. G., "Airbus A320 Side Stick and Fly By Wire - An Update: SAE Technical Paper 861801," 1986.

${ }^{9}$ Hegg, J. W., Smith, M. P., and Yount, L., "Sidestick Controllers for Advanced Aircraft Cockpits," Proceedings of the 11th AIAA/IEEE Digital Avionics Systems Conference, IEEE, New York, 1992, pp. 491-499.

${ }^{10}$ Grünhagen, W. v., Müllhäuser, M., Abildgaard, M., and Lantzsch, R., "Active Inceptors in FHS for Pilot Assistance Systems," 36th European Rotorcraft Forum, Paris, 2010.

${ }^{11}$ Taylor, A., Greenfield, A., and Sahasrabudhe, V., "The Development of Active Inceptor Systems and the Scope and Design Issues of Tactile Cueing Systems," American Helicopter Society 64th International Annual Forum, Alexandria, VA, 2008.

${ }^{12}$ U.S. Department of Transportation - Federal Aviation Administration (FAA), "Advisory Circular 120-71A: Standard Operating Procedures for Flight Deck Crewmembers (AC 120-71A)," U.S. Department of Transportation - Federal Aviation Administration (FAA), February 27th, 2003.

${ }^{13}$ U.S. Department of Transportation - Federal Aviation Administration (FAA), "Advisory Circular 120-51E: Crew Resource Management Training (AC 120-51E)," U.S. Department of Transportation - Federal Aviation Administration (FAA), January 22th, 2004.

${ }^{14}$ Uehara, A. F. and Niedermeier, D., "Limited Evaluation of the Influence of Coupled Sidesticks on the Pilot Monitoring's Awareness during Stall in Cruise," Proceedings of the AIAA Modeling and Simulation Technologies Conference, Vol. 1, 2013.

${ }^{15}$ Summers, L. G., Shannon, J. H., White, T. R., and Shiner, R. J., "Fly-by-Wire Sidestick Controller Evaluation: SAE Technical Paper Series \#871761," Proceedings of the SAE Aerospace Technology Conference and Exposition, 1987.

${ }^{16}$ Field, E. and Harris, D., "A comparative survey of the utility of cross-cockpit linkages and autoflight systems' backfeed to the control inceptors of commercial aircraft," Ergonomics, Vol. 41, No. 10, 1998, pp. 1462-1477.

${ }^{17}$ Rees, D. J. and Harris, D., "Effectiveness of Ab Initio Flight Training Using Either Linked or Unlinked Primary Axis Flight Controls," The International Journal of Aviation Psychology, Vol. 5, No. 3, 1995, pp. 291-304.

${ }^{18}$ Aircraft Accident Investigation Board (AAIB), "AAIB Bulletin No: 1/2013: Incident with the Airbus A319-111 G-EZFV at the London Luton Airport on February 14th, 2012," United Kingdom, 2013.

${ }^{19}$ Airbus, A320 Flight Crew Operating Manual: Systems Description, Vol. 1. Issue date: 01-Dec-2008, Toulouse - France, 2008.

${ }^{20}$ Sparko, A. L., Bürki-Cohen, J., and Go, T. H., "Transfer of Training from a Full-Flight Simulator vs. a High Level Flight Training Device with a Dynamic Seat," Proceedings of the AIAA Guidance, Navigation, and Control Conference, 2010.

${ }^{21}$ Endsley, M. R., "Design and Evaluation for Situation Awareness Enhancement," Proceedings of the 32nd Human Factors Society Annual Meeting, Vol. 32, 1988, pp. 97-101.

${ }^{22}$ Jones, D. G. and Endsley, M. R., "Sources of Situation Awareness Errors in Aviation," Aviation, Space, and Environmental Medicine Journal, Vol. 67, No. 6, 1996, pp. 507-512.

${ }^{23}$ Bureau d'Enquêtes et d'Analyses pour la sécurité de l'aviation civile (BEA), "Final Report on the accident on 1st June 2009 to the Airbus A330-203 registered F-GZCP operated by Air France flight AF447," France, July, 2012.

${ }^{24}$ Endsley, M. R., "Toward a theory of situation awareness in dynamic systems," Human Factors, Vol. 37, No. 1, 1995, pp. $32-64$.

${ }^{25}$ Airbus, A320 Flight Crew Operating Manual: Flight Operations, Vol. 3. Issue date: 01-Jun-2009, Toulouse - France, 2009.

${ }^{26}$ Endsley, M. R. and Garland, D. J., Situation Awareness Analysis and Measurement, Lawrence Erlbaum Associates, Inc, Mahwah, NJ, 2000.
}

16 of 16 